\title{
Orchestra ! : an Internet service for distributed musical sessions and collaborative music development and engineering
}

\section{P. Bussotti, F. Pirri}

Dept. Of Electronic Engineering, University of Florence

Via di S. Marta, 350100 Florence, Italy

telephone : 039-55-4796370 fax:039-55-488883

E-mail : bussotti@sunto.ing.unisi.it,fpirri@ing.unifi.it

\begin{abstract}
This paper proposes a platform for musical sessions distributed over the Internet and for music development and engineering. The system is based on a distributed software architecture and it's universally accessible through a simple http-client (a browser ). Orchestra! is a service that offers a common working environment and tools to the users, as well as a platform that provides a layer of common functionality for existing musical and groupware tools and for the development of new ones.

The main challenge of Orchestra! is to offer an useful and appealing service with little resources required on the client's side (i.e. a sound card and a browser) while providing an appreciable solution to the typical problems of collaborative realtime applications over the Internet, within the limits of this particular case and its requirements : musical group synchronisation.

As it is well known, packet-switched network's delays make impossible to reach a musical synchronisation among all members of a virtual group when each one is connected to the others. Nevertheless, by conjugating the ideas of hierarchical
\end{abstract}


rhythmic tree organisation ( from professional musical recording ) and of storeand-forward groupware systems, a stream communication model had been developed which is insensitive to delays. This should give an appreciable approximation of real-life musical sessions, with the remarkable plus-value of allowing distant people to play together, as well as providing a solution to the problem of rooms and equipment for group music.

Orchestra! provides basic functions for group music, such as the possibility to perform together across the net and to inhabit a collaborative environment where collective developing of music and multi-track recording is possible.

These can be taken as a basis for specialised applications in different fields: for example, in musical didactics a particular session arrangement would be required in order to fit the needs of teachers and classrooms, as well as in professional music security requirements could be fundamental, together with the needs for more sophisticated sound processing elements to be added as plug-ins of Orchestra!.

\section{Keywords \\ Group synchronisation, streams, group session, collaborative environment}

\section{INTRODUCTION}

In recent years the Internet has been rapidly evolving into a popular source of information and more and more useful and appealing services, in accord to its underpinning philosophy of a large and low-cost network. In this direction, the creation of the World Wide Web had first contributed to a more efficient information flowing through University Centres and, later, to the creation of a real common cultural heritage. Besides, shareware software has become a common resource in many anonymous ftp sites.

A fundamental step of this evolution has been the idea of distributed software architecture, a deeply innovative approach that, in its various forms, tends to free the local host from the actual possession of a specific application software by making its functionality available when required within a client-server paradigm.

The wide spreading of software models like Java, ActiveX and Corba clearly confirms this tendency, that's moving further towards even more radical solutions, like the so called network computers: they should even abandon the resident operative system for a distributed one. In this sense the application becomes an Internet service, the access to which can be left free or regulated by on-line registrations and certifications. The object-oriented or, at a deeper level, the component-oriented technology represents a powerful foundation for distributed architectures. The final user himself takes advantage of it because he 
can personalise its application by aggregating those components that best fit his needs.

Those techniques had also proved to be a very effective basis for the developing of collaborative applications, which is one main goal of telematics, both in the fields of distance learning and distance working.

Orchestra! gives its contribution in this direction, by developing a multimedia collaborative environment over a distributed software architecture.

\section{BACKGROUND}

Computer music is an example of how art and technology can co-operate to create new expressive forms. Beside the developing and spreading of MIDI keyboards and samplers, popular software like Cubase (1998) or CakeWalk (1998) had deeply changed the approach to music of groups, both at the professional and at the amateur's level.

Group music over the Internet is a natural extension of computer music and it's still a young field of research.

It encounters the critical problem of exchanging over a packet-switched network synchronous streams of data with an extremely low tolerance from delays. In fact, while playing in real-life, everyone gets an immediate feedback from himself and the others, and this permits the group synchronisation.

In other works about this argument, well documented in Paradiso (1997), like Spinning Disks of E. Metois or the Palette of Brain Opera at the Massachusetts Institute of Technology (MIT), based on the work of J. Yu, the focus has been put more on the creative and artistic aspect of working interactively with expertsystem generated sounds rather than on simulating a real-life musical group session (in this context we will relate to group session in the meaning of collective activity both in the real-life situation and in the Orchestra! framework).

Orchestra!, on the other hand, deals with audio streams (i.e. the voice, analogic instruments) as well as MIDI, and thus it suffers much more from network and processing latencies. That's why Orchestra! implements a specific strategy of masking the net to the user at the application level, by allowing only a cascadelike streaming among the members' hosts with each one mixing his contribution along the cascade, according to the natural levels of rhythmic priority.

Important contributions to Orchestra! design came also from recent literature about DVEs (Distributed Virtual Environments), especially for object-oriented techniques for manipulating common objects and for distributed system architectures for large numbers of users (Braham, 1997)(Barrus, 1997)(Rockwell, 1997)(Roehle, 1997)(Clark, 1997)(Anderson, 1997).

The lower level problem of intrinsic synchronisation of streamed audio ( the term intrinsic is here used to distinguish it from musical group synchronisation) 
has been approached using RTP (Real Time Protocol) technology background (Casney, 1996).

In this paper, we will refer loosely to connections in the meaning of point-to-point sessions, independently from the underlying transport protocol.

\section{USER NEED ANALYSIS AND ORCHESTRA! SERVICES}

From the analysis of needs of different typologies of users, it arises immediately that three main user profiles has to be considered, that is:

1. the professional user;

2. the user for didactic aims (teacher and student);

3. the amateur user.

The Orchestra! approach is to consider the third one (the amateur user) as a sort of basic user, in the sense that, if proper services were provided to him within a flexible object-oriented platform, then each other profile could be satisfied as well by specialising basic functions into more sophisticated ones and by adding new functions.

For example, the interaction management protocol system is held at a separate level from the actual function implementation. In such a way the peer-to-peer interaction protocol that is implemented in an amateurs' musical group, can be replaced by a new module performing a master-slave interaction protocol that better fits a music classroom's needs. Another example could be the professional user who can add security policies and more sophisticated signal processing systems as plug-ins of Orchestra!.

Referring to our target user, the focus has been put on the following points:

1. The application has to be accessible as an Internet service, and it should require the smallest amount of hardware resources on the client's side (in this case : a bi-directional sound-card and an interface to analogic/MIDI musical instruments or a microphone).

2. There has to be a high level of collaboration : every decision about a common resource has to be taken collectively after a discussion.

For this aim an audio chat-line has been provided, connecting all the member of the group among them. The access to the common resources is regulated by a privilege management system, and a privilege access protocol is implemented in order to obstruct undemocratic actions.

3. It must ensure a good quality of musical performance among people distributed over the Internet. That means that a solution (at least partial ) has to be found to the problem of musical synchronisation in presence of network's delay. 
4. Tools have to be provided for multi-track recording and sound engineering.

5. Music development is one of the most important and stimulating activity of a group, the one in which each individual brings his own ideas and confronts with the others in a constructive way. Therefore, adequate tools have to be provided to allow such an activity to be carried out within a net session.

6. All the tools have to be available also outside the collective session in such a way that everyone can work and practice individually with musical bases and tracks stored in his own hard disk.

7. External people could be allowed by the group to participate as an audience at the group's live performances.

Figure 1 illustrates a summarising scheme of Orchestra! services (user interface), classified as Logistic services, Interactive services and Playback and recording services (the scheme refers to our implementation using Java ${ }^{\mathrm{TM}}$ Applets, but it can be implemented in several other ways).

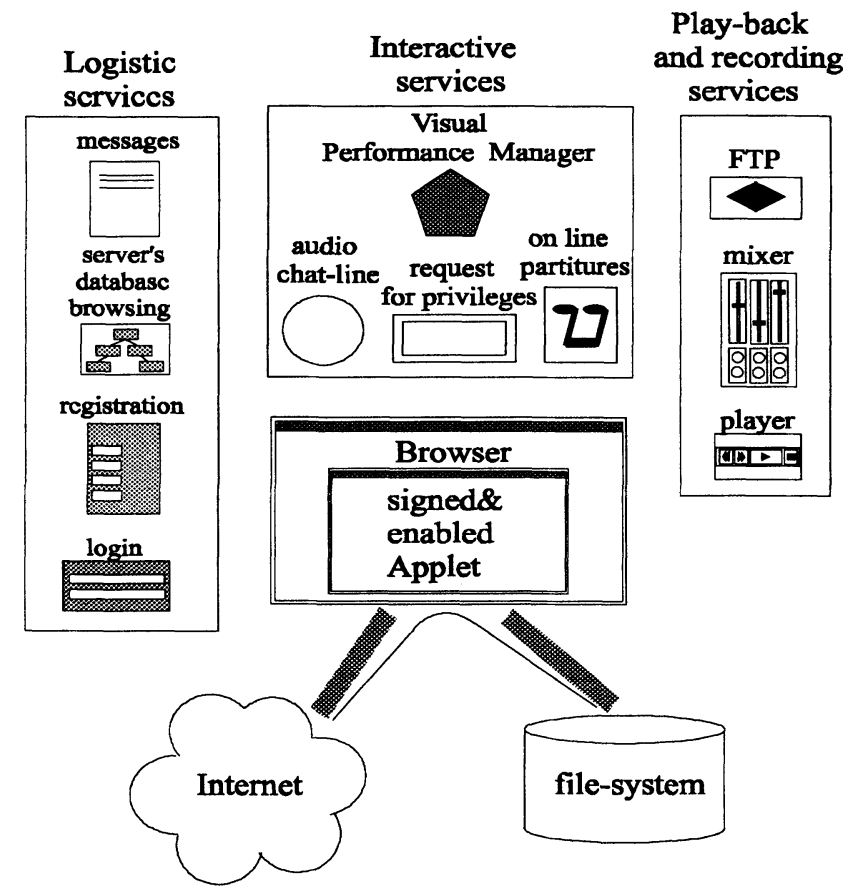

Figure 1 - Orchestra! services 
A more complete description of the Orchestra! platform will be given in the following sections :

- the system architecture;

- the hierarchical rhythmic tree approach;

- the collaborative environment;

- our prototype's implementation.

\subsection{The system architecture}

Orchestra! is based on a distributed software architecture that allows the application module to be accessed by multiple users from a common module server through a simple resident client application. This module is put in execution on the local machine and every host receives an identical copy of it : in this sense it is an Internet service, which frees the local host from actual possession of software, with economic advantages both for what concerns acquiring it and for memory occupation on the local hard disk. In particular, such an application will not suffer from versioning and the necessity of periodical software upgrading.

Orchestra! establishes a net connection among the dislocated hosts which belong to a common musical session, in order to implement the sharing of audio streams, musical tools and session data within a collaborative environment.

For this reasons, each single Orchestra! module has to be able to open connections towards other hosts, as well as to access local resources, as the filesystem for storing works, or for loading local libraries.

These capabilities are strongly dependent on the particular software architecture; for example, Java ${ }^{\mathrm{TM}}$ Applets require a special signing and privilegeenabling procedure to access resources outside its security sandbox, while ActiveX Controls don't suffer from any security restriction.

In order to implement an effective session management, Orchestra! uses a client-server paradigm at the application level by pairing an "Orchestra! Session Server" to the "Orchestra! Module Server" ( that is a Web Server in our Java implementation). The Orchestra! module is, then, the client side of the system : it creates a connection towards its session server as soon as it starts to run on the local machine.

The Orchestra! Session Server manages the sessions in a centralised way, by :

- keeping an updated database of groups and individuals registered to Orchestra!;

- keeping the current status (e.g. session on/off, play/idle, list of participants etc.) of each session;

- storing common group resources (as musical tracks, masters etc.); 
- allowing the access to common group resources in a collaborative way and regulating it by a privilege access protocol;

- keeping the table of hierarchical tree connections ( see paragraph 3.2 - the hierarchical tree approach) and allowing a collaborative modification of it through the Visual Performance Manager on the graphical user interface;

- notifying every change occurred in the session status to all the participants;

- providing a message mailbox for internal use of a group;

- tunneling and broadcasting audio streams.

Every action of the individual member on the system is broadcast to all the others. In particular, the audio chat-line uses the server to collect audio-streams from each one, mix them and then broadcast them to all the others.

In our implementation the server manages all the connections, acting as a broadcaster for chat-line audio streams and as a tunnel for the guide-stream of the Performance system (see paragraph: 3.2 - the hierarchical tree approach). This choice is in complete agreement with the idea of giving to the server the total control over the session, in such a way that the list of current participants and the related configuration of connections is made transparent to the user.

Orchestra! manages the connections' system at the Application Level of the OSI-Reference Model . It must be remarked, however, that multicast techniques could offer effective solutions, as well. When dealing with a large number of users the best solution could be a hybrid system with several servers communicating together.

\section{2 the hierarchical rhythmic tree approach}

As it is well known, the problem of delay, that's inherent to the nature of a packet-switched, non-homogeneous internetworking, gives a limit to the responsiveness of interactions, and thus to the quality of real-time collaborative environments. In order to focus on the real core functionality it has been decided not to deal with video data but only with audio, image and textual communication, which helps decreasing drastically the amount of required bandwidth. As it can be easily argued the critical point for an application that tries to reproduce a real-time interactive musical session is that it should reproduce the quasi-instantaneous propagation of sound within a small room: that's, in fact, the necessary condition for instrument players and vocalists to synchronise with each other. Of course, this is not achievable over the Internet, and the musical track of a player B that receives a track from a player A and synchronises himself on it will return to the player $A$ after twice the connection delay, that is unacceptable. Nevertheless, this work would try to show how even a very critical interactive situation (perhaps the most critical) could be approximated up to a certain degree of acceptance by a network application if proper strategies are adopted. 
The key idea comes from the techniques of professional musical recording, and, in particular, from the hierarchical rhythmic tree approach, where musical tracks are recorded in a sequence that starts from the instrument that has the highest rhythmic priority (i.e. the drums) down to lowest rhythmic priorities (i.e. bass guitar, rhythmic guitar, keyboards, soloists, vocalists).

In a professional recording context, this is used for ensuring a stable rhythmic basis for melodic instruments and voices that, for their congenital extremely variable performance (i.e. melody), are more subject to losing synchronisation.

In our context, we use this priority ordering to define a system of network connections among players : the drummer will play alone and communicate its captured musical stream to the bass guitar player who will synchronise himself on it and mix its contribution to the stream : it's evident that this can be done no matter how long the delay is. Than, in turns, he will communicate the digitally mixed stream (in which drums and bass are synchronised) to the rhythmic guitar player and so on. The lowest steps of the cascade (i.e. soloist, vocalist) will receive a stream that's acceptably rich of previous instruments' contributes, and this should give a sensation that's very close to the real-life session.

The constraint of this cascade system (which can be easily expanded into a tree structure) is that everyone receives the stream that comes from higher priority instruments and delivers it to lower priority ones : the fact that none gets a stream from someone he had previously sent a stream to, ensures that network latencies won't affect the correct functioning.

This uni-directional audio stream that passes through the cascade has the only function of allowing a right synchronisation of all the instruments and of creating an effective ensemble feeling ; this "guide stream" will occupy just one channel, because each new contribution is digitally mixed to the incoming stream. Meanwhile, each player produces also a copy of his own track that's kept separate from the mixed stream; this is marked in a proper way (by using RTP timestamping), in order to save time references for final tracks' mixing, and sent to a server's tracks' archive. It will keep a vectorial representation of each song in terms of separate tracks, which can be singularly edited or overwritten in a second time by an authorised person (e.g. each player can be authorised to perform I/O with his own tracks and read-only access to others' tracks). At the end of the cascade the guide stream can be discarded or broadcast to the audience : in each case, the final high-quality product is the master resulting from tracks' mixing.

A tracks' mixer is available, and the session member who have received a proper Mixer Operator token from the others is allowed to access all the tracks and mix them into a master, that is successively stored in the server space of the group as a read-only file. 
Figure 2 illustrates the basic guide-stream flowing through the cascade of hosts. Each of them receives it from the immediately upper priority instrument, adds it to its instrument's captured audio stream and sends the resultant stream to lower priority instruments. As far as the internal delay of the audio capture and playing system is a deterministic and known value, a synchronisation of the two stream is easily achieved ( for sack of clearness in fig. 1 the internal delay is considered to be null). At the same time, each instrument produces a copy of the track and stores it separately in the server's track-buffers. Of course, each track could be stored in the local hard disk and sent to the server at a second time, as well.

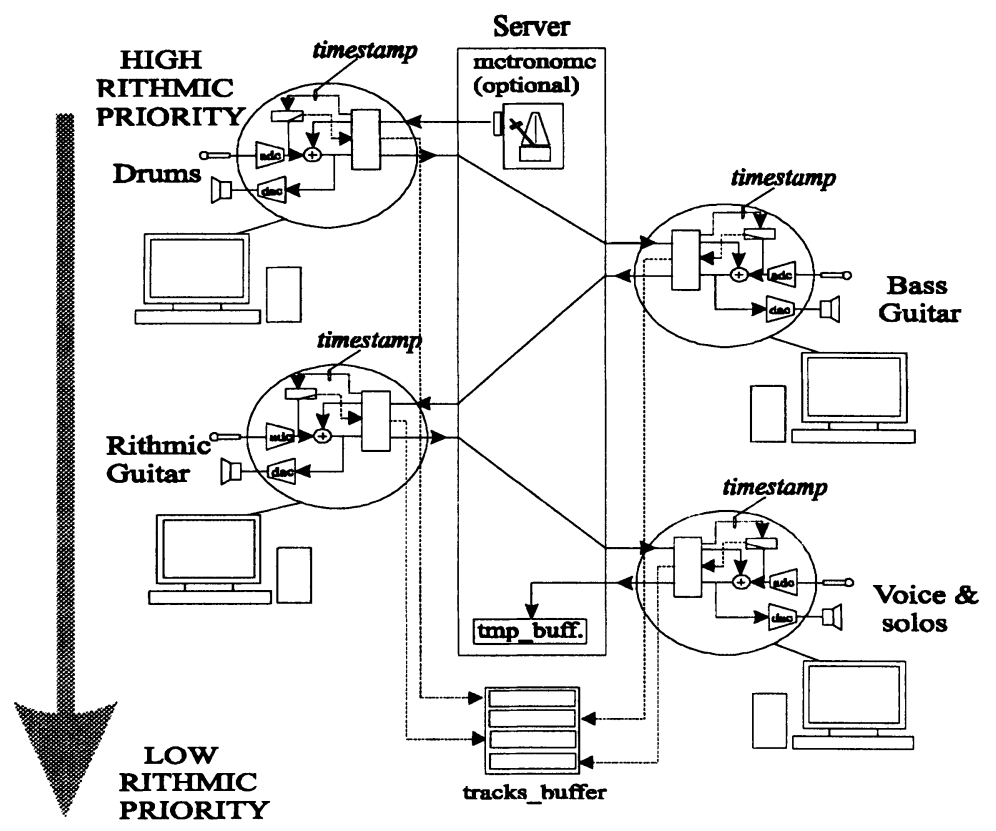

Figure 2 - guide-stream flowing through the Orchestra! cascade (tree)

It's convenient to use a low bit-rate waveform stream for the guide-stream, in order not to overload the network in time-sensitive operations, while the track should be a high-quality one or, when possible, a MIDI track (in this case the mixer has to implement MIDI format).

It could be argued that a system like this could work as well with a real store \&forward approach, where the whole block of waveform data is passed as a file through the cascade and, at each step a new instrument is added (this won't require anything more than FTP). This is true, but the choice of streaming realtime captured audio allows a much more satisfying ensemble sensation, for example when using the chat-line for interrupting the performance at an arbitrary point. 


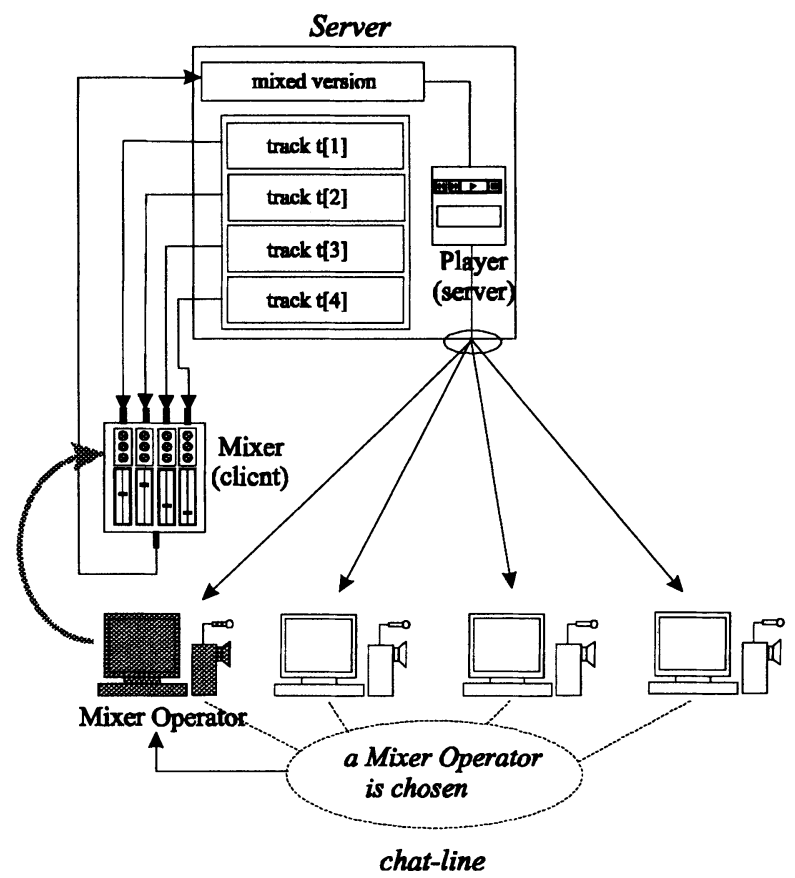

Figure 3 - tracks' mixing and broadcasting of the master.

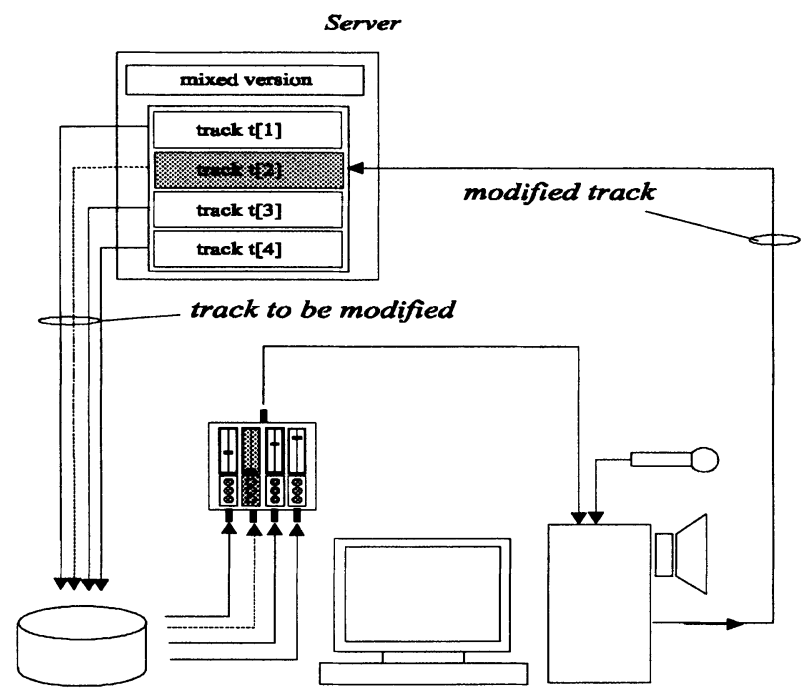

Figure 4 - Modifying of a track (stand-alone mode) 
Figure 3 illustrates the mixing phase and the playing of the master.

Figure 4 illustrates the single player that, out of the session (or in a stand-alone mode ), substitutes his own track with a new version.

To do this, he can access the session server to get all the song's tracks and store them locally, mix all the track except his own and then use this incomplete mixed version as the basis to play along with.

Then, he can overwrite his own track on the server and leave a message to the group for advising all the others about the replacing of his track.

The Orchestra! sub-system that manages the structure of the hierarchical rhythmic tree is named Performance System. It has a default way to organise currently present instruments in a tree structure, according to their rhythmic priority level and to the current list of session participants; in particular, if an instrument enters a session that's already begun, it'll be automatically inserted at its right place.

It must be noticed that, for the already mentioned reasons, if a node contains two or more parallel instruments (i.e. with the same priority) the system must decide which way the guide-stream will follow (two parallel streams can't be added together : one must be discarded), and this is done on a default basis which can be modified.

The Performance System plays also a key role in managing the external access to a session as Audience ( see paragraph 3, point 7 : User need analysis and Orchestra! services).

In fact, all the people who obtain an access to the session with an audience privilege is set by the Performance System at the end of the cascade (or of the main branch of the tree) in such a way that they can listen to the most complete version of the guide-stream.

Besides, the Performance System offers to the group a graphical interface that allows a visual editing of connections and a runtime monitoring of connections' status: the Visual Performance Manager.

Using this tool, after having received a proper Performance Manager token from the rest of the group, the default rhythmic tree can be modified in an arbitrary way, and this is particularly useful in cases like these :

- equal priority instruments can be arranged as consecutive;

- consecutive instruments can be arranged at the same hierarchic level;

- there can be set a different path for the guide-stream in cases of nodes with two or more instruments (i.e. proper tree structure instead of a cascade).

Another fundamental service which can be easily handled by the Visual Performance Manager is the music developing environment.

In fact situations like the following :

- one player presents his idea to the others; 
- two instruments ( or, in general, less than the total number ) practice to play together while the others are listening,

can be managed entering the Developing mode of the Visual Performance Manager. In this mode (as opposed to the Playing mode) not all instruments are assumed to be playing, and the active ones must be indicated by marking their icons on the graphical interface. All the unmarked instruments will be temporarily put at the end of the cascade, as listeners.

An evident problem of this system is that the ensemble feeling could be reasonably get by the only low priority instruments, which get a rich and articulate guide-stream from the upper rhythmic instruments. This could be obviate when the song is strictly synchronised by a metronome : in these cases a local guide-stream, containing only some lower tracks previously recorded, could be locally played with the drums or with the bass and then stopped inside them. This won't compromise anything if a correct metronome timing is used (notice that, in absence of a metronome, tempo and rhythmic variation can be decided by the drummer according to his improvisation ).

\subsection{The collaborative environment}

Orchestra! has been conceived as a collaborative environment where people could use telematic resources to perform all activities related to music in a realistic way. In particular, music is a field where the emotional factor is predominant over many other aspects and this has to be well analysed in order to meet a music player's expectations. Musical emotion arises from a mixture of acoustic satisfaction (that's why a high sound quality is required) and ensemble feeling (that 's why everyone have to feel in close contact with the others).

From personal experience in musical groups, I think that the pairing of an effective Performance System (with Playing/Developing modes) with an audio chat-line could be more important to a player than, for example, video data : music can be enjoyed with closed eyes.

The chat-line should be the main way to develop ideas, express comments and decide about common interests : that is, the real-life situation of the group that meet in a private music room has to be simulated as much as possible. Of course, the problem of network's delay will afflict the chat-line as well, but under certain limits, this will not compromise the discussion. In each case, the unregulated chat-line could be replaced, when required, by a regulated chatline, where some policy for sharing the audio channel is implemented.

Orchestra! defines common resources of a group like the current and old tracks, recording masters and so on, and offers to the user several tool to work with them in a really collaborative way. At the same time, the Performance 
System offers a Visual Interface for modifying its default configuration in a simple and intuitive way.

\subsection{Our prototype's implementation}

Our prototype of Orchestra! uses Java ${ }^{\mathrm{TM}}$ Applet technology (JavaAPI 1.0) for the implementation of the application module. In this case the module server is the web-server and the client application for getting it is a http-browser. This makes Orchestra! platform-independent. We opted for a Java implementation of Orchestra! mainly for the high level of integration of Java within the World Wide Web and for its independence from platforms : the choice of CORBA would have required a specific software installation on the client's side, while DCOM techniques (ActiveX) would have limited Orchestra! to Microsoft platforms.

We are creating an Orchestra! web-site where Orchestra! service is available after a registration phase. As far as JavaAPI 1.x do not provide any class for handling audio-streams (capturing, playing) native $C$ methods had been interfaced with the Java code in order to perform the mentioned tasks. This requires a dynamically linked library to be installed on the local machine and this is done at the first access to Orchestra! site by anonymous ftp.

Independence from platform is maintained by providing proper audio libraries for the most common operative systems. The access to local resources, like $\mathrm{read} / \mathrm{write}$ on the file system or loading a resident library could have been implemented by the use of Netscape ${ }^{T M}$ Capabilities Classes : this approach makes each local resource available to the Applet which is properly signed and authorised. On the other hand, this limits the choice of browsers to Netscape 4.0 or more recent versions.

\section{CONCLUSIONS}

As a conclusion, it must be stresses again the fact that Orchestra! is fundamentally a platform, a container that's flexible enough to support every different specialisation of its basic functionality, and to embed new components in its collaborative environment.

The main direction of our research will be, therefore:

- enhancement of Orchestra! performances;

- flexibility towards new audio formats;

- extensions of Orchestra in the directions of Music Distance Learning and Professional Music.

Another way we are expanding Orchestra! concerns the creation of a DVE where people can move along rooms and corridors : each active musical group will be assigned a room, and the visitor will listen to their music as soon as he enters that 
room. This could be a nice way to look for musical contacts with already formed groups in Orchestra!

\section{REFERENCES}

Cubase (1998), trademark of Steinberg Vetrieb GmbH. homepage : www.steinberg.de

CakeWalk (1998), trademark of Twelve Tone System, Inc. homepage : www.cakewalk.com

Paradiso, A.J. (1997) Electronic music: new ways to play. IEEE Spectrum, December 1997, volume 34, number 12, 18-30.

Braham, R. and Comerford, R. (1997) Sharing virtual worlds. IEEE Spectrum, March 1997, volume 34, number 3, 18-19.

Barrus, J.W. and Waters, R.C. (1997) The rise of shared virtual environments. IEEE Spectrum, March 1997, volume 34, number 3, 20-25.

Rockwell, R. (1997) An infrastructure for social software. IEEE Spectrum, March 1997, volume 34, number 3, 26-31.

Roehle, B. (1997) Channeling the data flood. IEEE Spectrum, March 1997, volume 34 , number 3, 32-38.

Clark, R.K. , Franceschini, R. and Reece, D. (1997) Synthetic soldiers. IEEE Spectrum, March 1997, volume 34, number 3, 39-45.

Anderson, D.B. and Casey, M.A. (1997) The sound dimension. IEEE Spectrum, March 1997, volume 34, number 3, 46-50.

Casner, S. , Frederick, R. , Jacobson, V. . Shulzrinne, H. (1996) RTP: A Transport Protocol for Real Time Applications. RFC 1889, January 1996.

\section{BIOGRAPHY}

Paolo Bussotti was born in Florence on July $20^{\text {th }}, 1966$. He received the Laurea (Dr. degree) from the University of Florence in 1996. He is currently a Doctorate student (II year) in Telematics at the University of Florence, where he is working on distributed software applications and systems, especially on the Java platform . 
He's also working on image and video processing and computer vision.

Franco Pirri was born in Livorno on May 14th, 1945. He received the Laurea (Dr. degree) from the University of Pisa in 1971. He joined the Dipartimento di Ingegneria Elettronica of the University of Firenze in 1973 and became Associate Professor of "Industrial Electronics" in 1982. He is currently Associate Professor of "Telematics" and of "Automatic Design of Electronic Circuits and Systems".

Franco Pirri is author of more than 75 papers, congress presentations and technical reports. His professional and academic experience has been focused on digital system design, with emphasis on communication networks, multimedia applications and application specific integrated circuits (ASIC).

$\mathrm{He}$ is member of the IEEE, the IEEE Computer Society and the IEEE Communication Society. 